\title{
Retrospective analysis of fosfomycin combinational therapy for sepsis caused by carbapenem-resistant Klebsiella pneumoniae
}

\author{
YUN LIAO $^{1 *}$, GUANG-HUI HU ${ }^{2 *}$, YUN-FEI XU $^{2}$, JIAN-PING CHE ${ }^{2}$, MING LUO $^{2}$, \\ HAI-MIN ZHANG ${ }^{2}$, BO PENG ${ }^{2}$, XU-DONG YAO ${ }^{2}$, JUN-HUA ZHENG ${ }^{2}$ and MIN LIU ${ }^{2}$ \\ Departments of ${ }^{1}$ Clinical Pharmacy and ${ }^{2}$ Urology, Shanghai Tenth People's Hospital, \\ Tongji University, Shanghai 200072, P.R. China
}

Received August 23, 2015; Accepted September 1, 2016

DOI: $10.3892 /$ etm.2017.4046

\begin{abstract}
The aim of the present study was to compare the efficacy and safety of fosfomycin combinational therapy with other antibiotics for the treatment of infections caused by carbapenem-resistant Klebsiella pneumoniae (CRKP). This retrospective cohort study examined 104 cases of sepsis caused by CRKP occurring between January 2012 and November 2014 in Shanghai Tenth People's Hospital. Three categories of patient outcome were assessed: Survival/mortality, duration of intensive care unit stays and duration of medical ventilation. Univariate ordinal analyses were adopted to evaluate the correlations between outcome and treatment. A total of 104 patients with physician-diagnosed CRKP were involved in the study. The overall mortality rate was $25.0 \%$. The majority of the infections $(84 ; 80.8 \%)$ were hospital acquired. Critical infections received more than one active antibiotic as therapy. Patients treated with fosfomycin combinational therapy were less likely to fail therapy (OR: $4.71,95 \%$ CI: 1.03-21.65, $\mathrm{P}=0.034)$ and tended to have a shorter duration of mechanical ventilation. Gender (OR: 4.35, 95\% CI: 1.08-3.60, P=0.037), history of chronic obstructive pulmonary disease (OR: 9.35, 95\% CI: 0.06-0.19, $\mathrm{P}=0.007)$ and peripheral catheter use $(\mathrm{OR}$ : $3.00,95 \%$ CI: $0.07-0.19, \mathrm{P}=0.002$ ) are risk factors for clinical outcome. Therefore, the use of fosfomycin combinational therapy for treatment of infection due to CRKP appears to be associated with improved survival rate.
\end{abstract}

Correspondence to: Dr Min Liu, Department of Urology, Shanghai Tenth People's Hospital, Tongji University, 301 Yangchang Road, Shanghai 200072, P.R. China

E-mail: kidneyliu@163.com

*Contributed equally

Key words: carbapenem-resistant Klebsiella pneumonia, sepsis, fosfomycin, meropenem

\section{Introduction}

Infections caused by carbapenem-resistant Klebsiella pneumoniae (CRKP) have been a serious problem due to limited therapeutic options all around the world (1). Few optimal therapy strategy are available for the infections, making its treatments extremely difficult and leading to poor therapeutic outcomes with the reported mortality rates ranging from $39-72 \%$ (2-4). The core issue of managing CRKP infection is to find an effective antibiotic regimen, as carbapenem resistance is often accompanied by resistance to other families of first-line antibiotics, such as beta-lactam inhibitors, quinolones and 3rd/4th generation cephalosporin. Optional antibiotics are usually limited to polymyxins, aminoglycosides, gentamicin, colistin and tigecycline $(5,6)$.

Recently, a study reported that treatment with tigecycline or an aminoglycoside can generate positive outcomes (7). In addition, there are reports indicating that the use of carbapenems in combination with other active agents could contribute to a lower mortality, particularly when the strains have low levels of in vitro resistance to those antimicrobials (8). Furthermore, a number of reports suggest that combinational therapies are often more effective than monotherapies $(9,10)$. Therefore, the goal of this retrospective study was to analyze the effect of combinational therapy of fosfomycin and carbapenemase on mortality from sepsis due to CRKP.

\section{Patients and methods}

Patients. The retrospective study was conducted in a teaching hospital of Tongji University (Shanghai, China). From January 2012 to November 2014. All CRKP isolates were collected from the database of the Microbiology Service of the Shanghai Tenth People's Hospital of Tongji University. The clinical records of patients with CRKP isolates were reviewed. The enrollment criteria were as follows: i) Clinical evidence of sepsis, severe sepsis or septic shock due to CRKP; ii) meropenem or imipenem were used within $72 \mathrm{~h}$ after CRKP cultured from urine, blood, phlegm or drainage liquid; iii) the administration of meropenem therapy lasted for at least $72 \mathrm{~h}$; and iv) $>18$ years old.

Admission to intensive care unit (ICU) was defined as a stay of $24 \mathrm{~h}$ before or after diagnosis of the severe sepsis. 
Predictors of the primary outcomes included age, sex, underlying diseases [diabetes mellitus, history of chronic obstructive pulmonary disease (COPD), heart failure, hepatic failure, renal failure, gastrointestinal surgery, solid malignancies, hematologic malignancies], special treatments (central venous catheter, peripheral catheter, foley catheter, Nasogastric tube, mechanical ventilation $\geq 3$ days, gastric acidity-lowering agents, immunosuppressive therapy, total parenteral nutrition), prior use of antibiotics and bacteremia. Clinical outcome contained length of ICU stay, mortality or cure, and duration of mechanical ventilation. The patients with recurrent CRKP isolated from the blood or urine were defined as microbiological failure at least 7 days after their index culture. Patients who had a negative culture were defined as treatment success.

Statistical analysis. Quantitative variables are expressed as the mean \pm standard deviation and qualitative variables are depicted as percentages of the group to which they belonged. Differences between patients treated with fosfomycin and meropenem and other antibiotics were analyzed by univariate logistic regression. Risk factors correlated with clinical outcome and microbiological failure was analyzed using stepwise multiple logistic regression analyses. The odds ratio (OR) was calculated with $95 \%$ confidence interval (CI). All statistical tests were two-tailed and utilized a 0.05 significance level. Analyses were performed using SPSS software, version 20.0 (IBM SPSS, Armonk, NY, USA).

\section{Results}

Clinical characteristics of the patients. A total of 104 unique cases of bacteremia with CRKP were identified during the experimental period. The demographics and clinical characteristics of the cases are shown in Table I. The ages of the 104 patients, 79 male and 25 female, ranged from 28 to 95 years, with a median of 67.2 years. The majority of the infections (84; $80.8 \%$ ) were hospital acquired. All but one patient had been admitted to the hospital within a year prior to the episode of bacteremia, with a majority of them $(87 ; 83.7 \%)$ having been admitted to an ICU. Patients that received ineffective empirical antimicrobial therapy before the susceptibility results became available are in the majority and 80 patients $(76.9 \%)$ received mechanical ventilation. The majority of patients had underlying diseases, had undergone invasive procedures, and had been admitted to ICUs or received medical services.

Antimicrobial susceptibility. Colistin exerted the highest susceptibility rate with $93.3 \%$ (Table II). Tigecycline and minocyline were active against $68(65.4 \%)$ and $79(76.5 \%)$ isolates, respectively. With regard to fosfomycin, 40 isolates $(38.5 \%)$ were susceptible, $54(51.9 \%)$ showed intermediate susceptibility and $10(9.6 \%)$ were resistant. A less marked susceptibility to amikacin $(28,26.9 \%)$ and gentamicin (14, $13.5 \%$ ) was observed.

Antibiotic treatment. All patients received targeted antibiotic treatment. Targeted treatment was optimal in 78 patients (75.0\%). The targeted antibiotics used were shown in Table III. A total of 10 patients were infected with a fosfomycin-resistant CRKP strain. This combination treatment group consisted of 24 patients $(23.1 \%)$ that were administered a dose of $12 \mathrm{mg} / 24 \mathrm{~h}$ and were not administered fosfomycin as monotherapy. In the fosfomycin combinational therapy group, 16 patients were administered meropenem ( 1 g every $8 \mathrm{~h}$ ) following with fosfomycin with the other 8 patients received fosfomycin, meropenem, tigecycline or (and) minocyline. Those patients received fosfomycin combination were less likely to fail therapy (OR: 4.71, 95\% CI: 1.03-21.65, $\mathrm{P}=0.030$ ). However, no difference between length of ICU stays and duration of mechanical ventilation was observed in these two groups (Table I). The primary characteristics of the patients who received fosfomycin combinational therapy are shown in Table IV. According to the result, the number of patients that underwent prior use of minoglycosides and carbapenems differed between the two groups. Compared with other regimens $(10.2 \pm 11.0$ vs. $13.0 \pm 11.2)$, fosfomycin combination group tends to have shorter duration of mechanical ventilation, however the difference did not display statistical significance. An interesting thing is that the age of fosfomycin combinational therapy group is older than other treatments group $(68.7 \pm 14.6$ vs. $62.0 \pm 18.3, \mathrm{P}=0.065)$, which might give us a hint that fosfomycin combinational therapy is safer and more suitable to be applicable to aged people. The fosfomycin combinational therapy group has an overall mortality of $8.3 \%$ with one case died of pneumonia and another died of the infection of the central nervous system (Table V).

Taking the difference in prior use of aminoglycosides into consideration, the effect of fosfomycin in combination with meropenem and with other regimens treatment was compared (Table VI). This combinational therapy group has 16 patients. Compared with other therapy, a significant difference was observed (Table VI, $\mathrm{P}=0.010$ ) when compared with treatment by using $3 \mathrm{rd} / 4$ th generation (monotherapy or combinational therapy).

Risk factors for mortality. Differences between cases of mortality and survival was observed using univariate analysis (Table I). Peripheral catheter and history of COPD have a negative influence on survival rates $(\mathrm{P}=0.01$ and $\mathrm{P}=0.016)$. Meanwhile, compared with female patients, male patients have a higher mortality. Fosfomycin combinational therapy (7.7 vs. $24.6 \%$ ) was associated with lower mortality (Table I). Compared with other targeted therapy, target treatment with fosfomycin exerted preponderance ( $\mathrm{P}=0.034$, OR: 4.71 [1.03-21.65]). The survival analysis performed using Kaplan-Meier curves showed that patients treated with fosfomycin combinational therapy had higher survival rates at 45 days after diagnosis (Fig. 1, log-rank test $\mathrm{t}=3.96, \mathrm{P}=0.047$ ). However, in a multivariate analysis, no correlation between fosfomycin combination and outcome was observed (Table VII, $\mathrm{P}=0.141$ ).

\section{Discussion}

CRKP has become a major hospital pathogen worldwide, and infections due to this organism have been associated with high mortality $(9,11-13)$. The increasing prevalence and the high mortality associated with the organism underscore the importance of effective antimicrobial therapy for these serious infections. However, optimal treatment for infections caused by CRKP has not yet been defined (14). 
Table I. Baseline characteristics of 104 patients with severe infection caused by carbapenem-resistant Klebsiella pneumoniae. Univariate analysis of factors associated with clinical outcome, $\mathrm{N}(\%)$.

\begin{tabular}{|c|c|c|c|c|c|}
\hline Demographic variables & Total (104) & Mortality (26) & Survivors (78) & P-value & OR $(95 \% \mathrm{CI})$ \\
\hline Age $($ mean $\pm \mathrm{SD})$ & $67.2 \pm 15.7$ & $68.4 \pm 15.5$ & $66.8 \pm 15.9$ & 0.641 & \\
\hline \multicolumn{6}{|l|}{ Gender } \\
\hline Male & $79(76.0)$ & $16(61.5)$ & $63(80.8)$ & 0.047 & $2.63(1.00-6.93)$ \\
\hline Female & $25(24.0)$ & $10(38.5)$ & $15(19.2)$ & & \\
\hline \multicolumn{6}{|l|}{ Underlying disease } \\
\hline Diabetes mellitus & $43(41.3)$ & $13(50.0)$ & $30(38.5)$ & 0.301 & $0.63(0.26-1.53)$ \\
\hline History of COPD & $29(27.9)$ & $12(46.2)$ & $17(21.8)$ & 0.016 & $0.32(0.13-0.83)$ \\
\hline Heart failure & $24(23.1)$ & $8(30.8)$ & $16(20.5)$ & 0.282 & $0.58(0.21-1.58)$ \\
\hline Hepatic failure & 7 (1.9) & $3(11.5)$ & $4(5.1)$ & 0.363 & $0.41(0.09-1.99)$ \\
\hline Renal failure & $24(23.1)$ & $8(30.8)$ & $16(20.5)$ & 0.293 & $0.58(0.21-1.58)$ \\
\hline Gastrointestinal surgery & $9(8.7)$ & $3(11.5)$ & $6(7.7)$ & 0.687 & $0.64(0.15-2.76)$ \\
\hline Solid malignancies & $14(13.5)$ & $4(15.4)$ & $10(12.8)$ & 0.746 & $0.81(0.23-2.84)$ \\
\hline Hematologic malignancies & $0(0)$ & $0(0)$ & $0(0)$ & & \\
\hline \multicolumn{6}{|l|}{ Special treatments total } \\
\hline Central venous catheter & $72(69.2)$ & $17(65.4)$ & $55(70.5)$ & 0.624 & $1.27(0.49-3.25)$ \\
\hline Peripheral catheter & $43(41.3)$ & $18(69.2)$ & $25(32.1)$ & 0.001 & $0.21(0.08-0.55)$ \\
\hline Foley catheter & $86(82.7)$ & $21(80.8)$ & $65(83.3)$ & 0.765 & $1.19(0.38-3.73)$ \\
\hline Nasogastric tube & $78(75.0)$ & $22(84.6)$ & $56(71.8)$ & 0.295 & $0.46(0.14-1.50)$ \\
\hline Mechanical ventilation $\geq 3$ days & $70(68.0)$ & $19(73.1)$ & $51(66.2)$ & 0.518 & $0.72(0.27-1.94)$ \\
\hline Gastric acidity-lowering agents & $98(94.2)$ & $25(96.2)$ & $73(93.6)$ & 1.00 & $0.58(0.07-5.24)$ \\
\hline Immunosuppressive therapy & $29(27.9)$ & $4(15.4)$ & $25(32.1)$ & 0.132 & $2.59(0.81-8.33)$ \\
\hline Total parenteral nutrition & $44(42.3)$ & $10(38.5)$ & $34(43.6)$ & 0.647 & $1.24(0.50-3.06)$ \\
\hline \multicolumn{6}{|l|}{ Prior use of antibiotics } \\
\hline$\beta$-lactam inhibitors & $52(50.0)$ & $17(65.4)$ & $35(44.9)$ & 0.070 & $0.43(0.17-1.08)$ \\
\hline Quinolones & $42(40.4)$ & $14(53.8)$ & $28(35.9)$ & 0.106 & $0.48(0.20-1.18)$ \\
\hline $\begin{array}{l}\text { 3rd/4th Generation } \\
\text { cephalosporin }\end{array}$ & $72(69.2)$ & $17(65.4)$ & $55(70.5)$ & 0.624 & $1.27(0.49-3.25)$ \\
\hline Aminoglycosides & $21(20.6)$ & $2(7.7)$ & $19(25.0)$ & 0.060 & $4.0(0.86-18.53)$ \\
\hline Fosfomycin & $6(5.8)$ & $0(0)$ & $6(7.7)$ & 0.333 & $0.74(0.65-0.83)$ \\
\hline Imipenem & $14(13.5)$ & $1(3.8)$ & $13(16.7)$ & 0.097 & $5.00(0.62-40.25)$ \\
\hline \multicolumn{6}{|l|}{ Type of infection } \\
\hline CAP & $28(26.9)$ & $6(23.1)$ & $22(28.2)$ & 0.610 & $1.31(0.46-3.69)$ \\
\hline HAP & $84(80.8)$ & $21(80.8)$ & $63(80.8)$ & 1.000 & $1.00(0.32-3.08)$ \\
\hline Urinary tract infection & $17(16.3)$ & $5(19.2)$ & $12(15.4)$ & 0.646 & $0.76(0.24-2.42)$ \\
\hline Surgical site infection & $11(10.6)$ & $2(7.7)$ & $9(11.5)$ & 0.581 & $1.57(0.32-7.76)$ \\
\hline Intra-abdominal infecton & $4(3.8)$ & $1(3.8)$ & $3(3.8)$ & 1.000 & $1.04(1.00-1.08)$ \\
\hline Primary bacteraemia & $9(8.7)$ & $3(11.5)$ & $6(7.7)$ & 0.546 & $0.64(0.15-2.76)$ \\
\hline $\begin{array}{l}\text { Central venous catheter } \\
\text { bacteraemia }\end{array}$ & $0(0)$ & $0(0)$ & $0(0)$ & & \\
\hline Ventilator associated pneumonia & $1(1.0)$ & $0(0)$ & $1(1.3)$ & 1.000 & $0.75(067-0.83)$ \\
\hline \multicolumn{6}{|l|}{ Targeted treatment } \\
\hline Monotherapy & $32(30.8)$ & $11(42.3)$ & $21(26.9)$ & 0.141 & $0.50(0.20-1.27)$ \\
\hline Combination therapy & $72(69.2)$ & $15(57.7)$ & $57(73.1)$ & & \\
\hline Fosfomycin combination & $24(23.1)$ & $2(7.7)$ & $22(28.2)$ & 0.034 & $4.71(1.03-21.65)$ \\
\hline Other treatment regimens & $65(61.9)$ & $16(24.6)$ & $49(75.4)$ & & \\
\hline Length of ICU stays & & $15.2 \pm 10.5$ & $17.6 \pm 12.2$ & 0.355 & \\
\hline Duration of mechanical ventilation & & $10.7 \pm 10.6$ & $10.9 \pm 10.9$ & 0.958 & \\
\hline
\end{tabular}

Values in bold are statistically significant. SD, standard deviation; COPD, chronic obstructive pulmonary disorder; CAP, community-acquired pneumonia; HAP, hospital-acquired pneumonia; ICU, intensive care unit; OR, odds ratio; CI, confidence interval. 
Table II. Antimicrobial susceptibility test result of 104 patients with severe infection caused by carbapenem-resistant Klebsiella pneumoniae.

\begin{tabular}{lccc}
\hline Drug & Sensitive & Intermediary & Resistance \\
\hline Tigecycline & $68(65.4)$ & $1(1.0)$ & $35(33.7)$ \\
Minocyline & $79(76.0)$ & $17(16.3)$ & $8(7.7)$ \\
Colistin & $97(93.3)$ & $0(0)$ & $7(6.7)$ \\
Gentamicin & $14(13.5)$ & $0(0)$ & $91(86.5)$ \\
Amikacin & $28(26.9)$ & $0(0)$ & $76(73.1)$ \\
Meropenem & $1(1.00$ & $0(0)$ & $103(99.0)$ \\
Imipenem & $1(1.00$ & $0(0)$ & $103(99.0)$ \\
Ertapenem & $1(1.00$ & $0(0)$ & $103(99.0)$ \\
Cefepime & $9(8.7)$ & $0(0)$ & $95(91.3)$ \\
Fosfomycin & $40(38.5)$ & $54(51.9)$ & $10(9.6)$ \\
\hline
\end{tabular}

The present retrospective study involved 104 unique patients with bacteremia due to CRKP with overall mortality rate of $25 \%$, which is substantially lower than the rates reported for CRKP in previous studies $(11,15,16)$. Alternatively, the difference may be resulted from unrecognized confounding variables. Nonetheless, the mortality still remains considerably higher than for bacteremia due to CRKP (17).

We identified gender, peripheral catheter and history of COPD as independent clinical risk factors for mortality. The present study demonstrated that survival in patients with CRKP was significantly improved when combinational therapy rather than monotherapy was administered. Fosfomycin combinational therapy is successful in the present study compared with other monotherapy and combination regimens. Alternatively, as monotherapy, fosfomycin could drive resistance develops rapidly (18). In the present study, no patients were included that received fosfomycin monotherapy. Fosfomycin is a broad spectrum antibiotic that inhibits peptidoglycan synthesis (19). Fosfomycin inhibits bacterial cell wall biogenesis by inactivating the enzyme UDP-N-acetylglucosamine-3-enolpyruvyltransferase which is also known as MurA (20). This enzyme is able to catalyze the committed step in peptidoglycan biosynthesis, namely the ligation of phosphoenol pyruvate to the 3'-hydroxyl group of UDP-N-acetyl glucosamine (20), which may provide a route for meropenem to enter the bacteria. It can be administered in combination with some other antimicrobial agents, since there is low level of or no cross resistance (21). This agent is well tolerated and has a few side effects. It has an increased role as a therapeutic option against multidrug-resistant Enterobacteriaceae $(19,20,22,23)$. Cumulative susceptibility of fosfomycin against ESBL positive Enterobacteriaceae is reported as $87.7 \%$ according to CLSI criteria (24). However, lower susceptibility rates $(<50 \%)$ was showed in some studies, and in a study this agent was shown to be active against only $50 \%$ of Klebsiella $(22,25)$.

Qureshi et al (3) reported that either colistin-polymyxin B or tigecycline in combination with a carbapenem appeared to be effective. These combination regimens were more successful than monotherapy with either colistin-polymyxin B or tigecycline, even when in vitro testing confirmed suscepti-
Table III. Target antibiotics used in 104 patients with severe infection caused by carbapenem-resistant Klebsiella pneumoniae, N (\%).

\begin{tabular}{|c|c|c|c|}
\hline Target antibiotic & Total & Mortality & Survivors \\
\hline Target monotherapy & $32(30.8)$ & $11(42.3)$ & $21(26.9)$ \\
\hline Pipercillin/sulbactam & $4(12.5)$ & $1(9.1)$ & $3(14.3)$ \\
\hline Aminoglycosides & $5(15.6)$ & $1(9.1)$ & $4(19.0)$ \\
\hline $\begin{array}{l}\text { 3rd/4th Generation } \\
\text { cephalosporin }\end{array}$ & $13(40.6)$ & $6(54.5)$ & $7(33.3)$ \\
\hline Quinolones & $4(12.5)$ & $0(0)$ & $0(0)$ \\
\hline Meropenem & $7(21.9)$ & $3(27.3)$ & $4(19.0)$ \\
\hline Imipenem & $1(3.1)$ & $0(0)$ & $1(4.8)$ \\
\hline Tigecycline & $1(3.1)$ & $0(0)$ & $1(4.8)$ \\
\hline Minocyline & $1(3.1)$ & $0(0)$ & $1(4.8)$ \\
\hline Target combination therapy & $72(69.2)$ & $15(57.7)$ & $57(73.1)$ \\
\hline Fosfomycin+meropenem & $16(19.4)$ & $1(6.7)$ & $15(24.6)$ \\
\hline $\begin{array}{l}\text { Fosfomycin+meropenem+ } \\
\text { Tigecycline/minocyline/ } \\
\text { amikacin }\end{array}$ & $8(11.1)$ & $1(6.7)$ & $7(12.2)$ \\
\hline Tigecycline+meropenem & $4(5.6)$ & $1(5.3)$ & $3(1.2)$ \\
\hline $\begin{array}{l}\text { Tigecycline+meropenem+ } \\
\text { amikacin }\end{array}$ & $6(8.3)$ & $1(5.3)$ & $5(8.8)$ \\
\hline Tigecycline+minocyline & $3(4.2)$ & $0(0)$ & $3(5.3)$ \\
\hline $\begin{array}{l}\text { Tigecycline+minocyline+ } \\
\text { meropenem }\end{array}$ & $1(1.4)$ & $0(0)$ & $1(1.8)$ \\
\hline Minocyline+carbapenems & $4(5.6)$ & $2(13.3)$ & $2(3.5)$ \\
\hline $\begin{array}{l}\text { Minocyline+pipercillin/ } \\
\text { sulbactam }\end{array}$ & $1(1.4)$ & $1(5.3)$ & $0(0)$ \\
\hline Gentamicin+meropenem & $2(2.8)$ & $0(0)$ & $2(3.5)$ \\
\hline Amikacin+meropenem & $3(4.2)$ & $0(0)$ & $3(5.3)$ \\
\hline Amikacin+cefepime & $3(4.2)$ & $0(0)$ & $3(5.3)$ \\
\hline $\begin{array}{l}\text { Amikacin+pipercillin/ } \\
\text { sulbactam }\end{array}$ & $1(1.4)$ & $0(0)$ & $1(1.8)$ \\
\hline $\begin{array}{l}\text { Cefepime+amoxicillin/ } \\
\text { sulbactam }\end{array}$ & $1(1.4)$ & $0(0)$ & $1(1.8)$ \\
\hline $\begin{array}{l}\text { Cefepime+pipercillin/ } \\
\text { sulbactam }\end{array}$ & $1(1.4)$ & $0(0)$ & $1(1.8)$ \\
\hline Cefepime+sulfamethoxazole & $2(2.8)$ & $2(13.3)$ & $0(0)$ \\
\hline Cefepime+aztreonam & $1(1.4)$ & $0(0)$ & $1(1.8)$ \\
\hline $\begin{array}{l}\text { Cefoperazone/sulbactam+ } \\
\text { carbapenems }\end{array}$ & $4(5.6)$ & $0(0)$ & $4(7.0)$ \\
\hline $\begin{array}{l}\text { Cefoperazone/sulbactam+ } \\
\text { quinolones }\end{array}$ & $2(2.8)$ & $1(5.3)$ & $1(1.8)$ \\
\hline Carbapenems+quinolones & $5(6.9)$ & $3(20.0)$ & $2(3.5)$ \\
\hline $\begin{array}{l}\text { Pipercillin/sulbactam+ } \\
\text { carbapenems }\end{array}$ & $4(5.6)$ & $2(13.3)$ & $2(3.5)$ \\
\hline
\end{tabular}

bility to the respective antimicrobials. While the mechanisms underlying the effectiveness of these combinations are not known, synergistic activity between carbapenems and colistin has been observed in vitro among their CRKP isolates, which is consistent with the clinical observation, at least for this particular combination. 
Table IV. Analysis of clinical variables in 24 patients that received fosfomycin combination therapy, N (\%).

\begin{tabular}{|c|c|c|c|c|c|}
\hline Demographic variable & Total & $\begin{array}{c}\text { Fosfomycin } \\
\text { combination }(n=24)\end{array}$ & $\begin{array}{l}\text { Other regimens } \\
\qquad(\mathrm{n}=80)\end{array}$ & $\mathrm{P}$ & OR $(95 \% \mathrm{CI})$ \\
\hline Age (mean \pm SD) & $67.2 \pm 15.7$ & $68.7 \pm 14.6$ & $62.0 \pm 18.3$ & 0.065 & \\
\hline \multicolumn{6}{|l|}{ Gender } \\
\hline Male & $79(76.0)$ & $20(83.3)$ & $59(73.8)$ & 0.422 & $1.78(0.55-5.81)$ \\
\hline Female & $25(24.0)$ & $4(16.7)$ & $21(26.2)$ & & \\
\hline \multicolumn{6}{|l|}{ Underlying disease } \\
\hline Diabetes mellitus & $43(41.3)$ & $6(25.0)$ & $37(46.2)$ & 0.064 & $0.39(0.14-1.08)$ \\
\hline History of COPD & $29(27.9)$ & $4(16.7)$ & $25(31.2)$ & 0.201 & $0.44(0.14-1.42)$ \\
\hline Heart failure & $24(23.1)$ & $2(8.3)$ & $22(27.5)$ & 0.057 & $0.24(0.05-1.11)$ \\
\hline Hepatic failure & $7(6.0)$ & $0(0)$ & $7(8.8)$ & 0.197 & $0.75(0.67-0.84)$ \\
\hline Renal failure & $24(23.1)$ & $3(12.5)$ & $21(26.2)$ & 0.268 & $0.40(0.11-1.49)$ \\
\hline Gastrointestinal surgery & $9(8.7)$ & $1(4.2)$ & $8(10.0)$ & 0.681 & $0.39(0.05-3.30)$ \\
\hline Solid malignancies & $14(13.5)$ & $2(8.3)$ & $12(15.0)$ & 0.513 & $0.52(0.11-2.48)$ \\
\hline Hematologic malignancies & $0(0)$ & $0(0)$ & $0(0)$ & & \\
\hline \multicolumn{6}{|l|}{ Special treatments total } \\
\hline Central venous catheter & $72(69.2)$ & $17(70.8)$ & $55(68.8)$ & 0.846 & $1.10(0.41-3.00)$ \\
\hline Peripheral catheter & $43(41.3)$ & $9(37.5)$ & $34(42.5)$ & 0.663 & $0.81(0.32-2.07)$ \\
\hline Foley catheter & $86(82.7)$ & $18(75.0)$ & $68(85.0)$ & 0.256 & $0.53(0.18-1.61)$ \\
\hline Nasogastric tube & $78(75.0)$ & $17(70.8)$ & $61(76.2)$ & 0.591 & $0.76(0.27-2.10)$ \\
\hline Mechanical ventilation $\geq 3$ days & $70(68.0)$ & $15(62.5)$ & $55(68.0)$ & 0.513 & $0.73(0.28-1.90)$ \\
\hline Gastric acidity-lowering agents & $98(94.2)$ & $23(95.8)$ & $75(93.8)$ & 1.00 & $1.53(0.17-13.80)$ \\
\hline Immunosuppressive therapy & $29(27.9)$ & $10(41.7)$ & $19(43.7)$ & 0.086 & $2.29(0.88-6.00)$ \\
\hline Total parenteral nutrition & $44(42.3)$ & $13(54.2)$ & $31(38.8)$ & 0.139 & $1.80(0.74-4.69)$ \\
\hline \multicolumn{6}{|l|}{ Prior use of antibiotics } \\
\hline$\beta$-lactam inhibitors & $52(50.0)$ & $15(62.5)$ & $37(46.2)$ & 0.163 & $1.94(0.76-4.94)$ \\
\hline Quinolones & $42(40.4)$ & $7(29.2)$ & $35(43.8)$ & 0.202 & $0.53(0.20-1.42)$ \\
\hline 3rd/4th Generation cephalosporin & $58(55.2)$ & $23(57.5)$ & $35(53.8)$ & 0.715 & $1.16(0.52-2.57)$ \\
\hline Aminoglycosides & $21(20.6)$ & $10(45.5)$ & $11(13.8)$ & 0.001 & $5.23(1.82-15.00)$ \\
\hline Fosfomycin & $6(5.8)$ & $3(12.5)$ & $3(3.8)$ & 0.134 & $3.67(0.6-19.51)$ \\
\hline Carbapenems & $14(13.5)$ & $8(33.3)$ & $6(7.5)$ & 0.001 & $6.17(1.88-20.24)$ \\
\hline \multicolumn{6}{|l|}{ Type of infection } \\
\hline CAP & $28(25.7)$ & $6(22.5)$ & $22(27.7)$ & 0.809 & $0.88(0.31-2.50)$ \\
\hline HAP & $84(80.8)$ & $19(79.2)$ & $65(81.2)$ & 0.820 & $0.88(0.28-2.73)$ \\
\hline Primary bacteraemia & $9(8.7)$ & $4(16.7)$ & $5(6.2)$ & 0.206 & $3.00(0.74-12.22)$ \\
\hline Central venous catheter bacteraemia & $0(0)$ & $0(0)$ & $0(0)$ & & \\
\hline Ventilator associated pneumonia & $1(1.0)$ & $1(4.2)$ & $0(0)$ & 0.381 & $1.03(0.98-1.08)$ \\
\hline Urinary tract infection & $17(16.3)$ & $3(12.5)$ & $14(17.5)$ & 0.756 & $0.67(0.18-2.57)$ \\
\hline Surgical site infection & $11(10.6)$ & $4(16.7)$ & $7(8.8)$ & 0.273 & $2.09(0.56-7.84)$ \\
\hline Intra-abdominal infecton & $4(3.8)$ & $1(4.2)$ & $3(3.8)$ & 1.000 & $1.12(0.11-11.25)$ \\
\hline Length of ICU stays & & $17.4 \pm 11.1$ & $15.6 \pm 14.2$ & 0.515 & \\
\hline Duration of mechanical ventilation & & $10.2 \pm 11.0$ & $13.0 \pm 11.2$ & 0.264 & \\
\hline
\end{tabular}

Values in bold are statistically significant. SD, standard deviation; COPD, chronic obstructive pulmonary disorder; CAP, community-acquired pneumonia; HAP, hospital-acquired pneumonia; ICU, intensive care unit.

It is considered that treatment with fosfomycin is free from the nephrotoxicity that characterizes treatment with aminoglycosides. A previous animal study demonstrated that fosfomycin has a protective effect against nephrotoxicity as a result of treatment with aminoglycosides by inhibiting aminoglycoside-induced histamine release from mast-cell destruction (26).

The present study is limited by its observational nature and relatively small number of cases, since we limited the analysis to cases with bacteremia only. In conclusion, mortality associ- 
Table V. Characteristics of patients with severe infection caused by carbapenem-resistant Klebsiella pneumoniae treated with targeted fosfomycin combination; $\mathrm{N}=24$.

\begin{tabular}{lr}
\hline Characteristic & $\mathrm{N}(\%)$ \\
\hline Overall mortality & $2 / 24(8.3)$ \\
Mortality by infection site & $1 / 24$ \\
Pneumonia & $0 / 24$ \\
Urinary tract infection & $0 / 24$ \\
Surgical wound infection & $0 / 24$ \\
Intra-abdominal infection & $0 / 24$ \\
Catheter-related bacteraemia & $1 / 24(4.2)$ \\
Infection of the CNS & $16 / 24(66.7)$ \\
Treatment regimens & $8 / 24(33.3)$ \\
Fosfomycin+meropenem & \\
Fosfomycin+meropenem+tigecycline/ & \\
minocyline/amikacin & \\
\hline
\end{tabular}

CNS, central nervous system.

Table VI. Comparison of patients treated with fosfomycin combination therapy against other treatment regimens, N (\%).

\begin{tabular}{|c|c|c|c|c|c|}
\hline Treatment & Total (104) & Mortality (26) & Survival (78) & $\mathrm{P}$ & OR $(95 \% \mathrm{CI})$ \\
\hline Fosfomycin+meropenem & $16(15.4)$ & $1(3.8)$ & $22(28.2)$ & & \\
\hline \multicolumn{6}{|c|}{ Monotherpy and in combination } \\
\hline Tigecycline/minocyline & $21(20.2)$ & $2(7.7)$ & $19(24.4)$ & $0.371^{\mathrm{a}}$ & $0.26(0.04-1.50)$ \\
\hline Aminoglycosides & $12(11.5)$ & $1(3.8)$ & $11(14.1)$ & $1.000^{\mathrm{a}}$ & $0.73(0.04-13.05)$ \\
\hline$\beta$-lactam inhibitors & $8(7.7)$ & $2(7.7)$ & $6(7.7)$ & $0.249^{\mathrm{a}}$ & $0.14(0.03-0.75)$ \\
\hline Quinolones & $8(7.7)$ & $2(7.7)$ & $6(7.7)$ & $0.249^{\mathrm{a}}$ & $0.20(0.02-2.64)$ \\
\hline 3rd/4th Generation & $21(20.2)$ & $10(38.5)$ & $11(14.1)$ & $0.010^{a, b}$ & $0.07(0.01-0.66)$ \\
\hline
\end{tabular}

Carbapenems+quinolones a compared with fosfomycin and meropenem group. ${ }^{\mathrm{b}}$ Other combination therapy including: Cefepime or cefoperazone/sulbactam in combination with carbapenems/ $\beta$-lactam inhibitors. Values in bold are statistically significant.

Table VII. Multivariate models of risk factors for mortality in patients with carbapenem-resistant Klebsiella pneumoniae sepsis.

\begin{tabular}{|c|c|c|c|c|c|c|}
\hline \multirow[b]{2}{*}{ Variable } & \multicolumn{2}{|c|}{$\begin{array}{c}\text { Unstandardized } \\
\text { Coefficients }\end{array}$} & \multicolumn{2}{|c|}{$\begin{array}{c}\text { Standardized } \\
\text { Coefficients }\end{array}$} & \multicolumn{2}{|c|}{$95 \% \mathrm{CI}$} \\
\hline & $\mathrm{B}$ & Std. Error & Beta & Sig & Up & Down \\
\hline (Constant) & 1.371 & 0.592 & 5.369 & 0.020 & & \\
\hline Gender & 1.279 & 0.614 & 4.349 & 0.037 & 3.594 & 1.080 \\
\hline History of COPD & -1.644 & 0.608 & 7.311 & 0.007 & 0.193 & 0.059 \\
\hline Peripheral catheter & -1.660 & 0.543 & 9.345 & 0.002 & 0.190 & 0.066 \\
\hline Fosfomycin combination & 1.202 & 0.817 & 2.167 & 0.141 & 3.326 & 0.671 \\
\hline
\end{tabular}

COPD, chronic obstructive pulmonary disorder.

ated with bacteremia due to CRKP continues to be high. The use of combinational therapy, with fosfomycin combinational therapy in particular, seems to have a survival benefit in this critically ill population.

\section{Acknowledgements}

This study was partially supported by grants from the National Natural Science Foundation of China (grant nos. 81403029, 


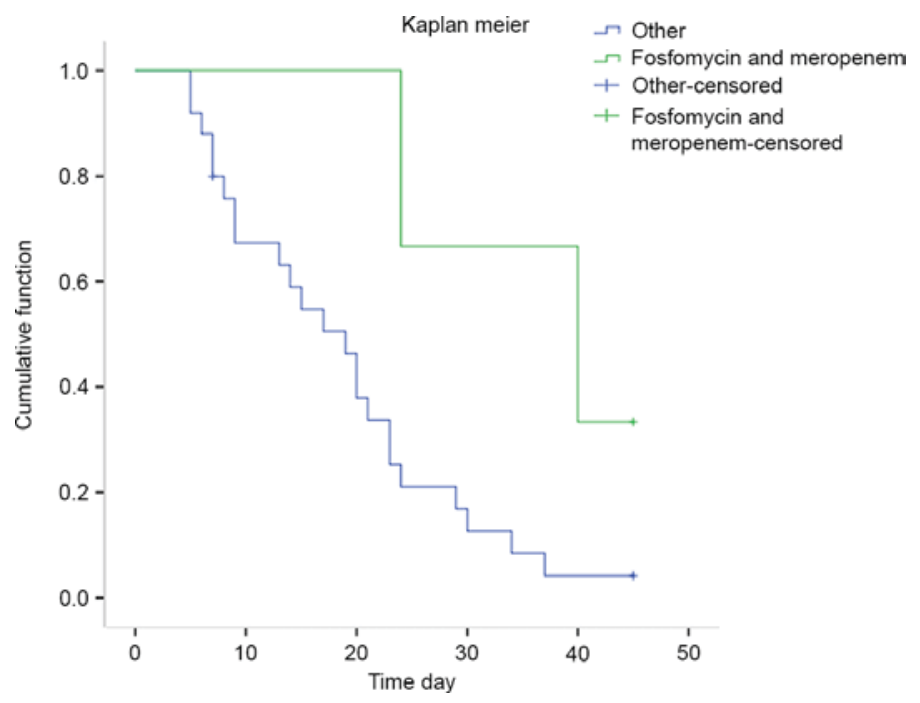

Figure 1. Kaplan-Meier curves showing the impact of targeted treatment with fosfomycin on survival at 45 days in patients with severe infection caused by carbapenem-resistant and colistin-resistant Klebsiella pneumonia (log-rank test, t=3.96, $\mathrm{P}=0.047$ ).

\section{1 and 81270831).}

\section{References}

1. Tzouvelekis LS, Markogiannakis A, Psichogiou M, Tassios PT and Daikos GL: Carbapenemases in Klebsiella pneumoniae and other Enterobacteriaceae: An evolving crisis of global dimensions. Clin Microbiol Rev 25: 682-707, 2012.

2. van Duin D, Kaye KS, Neuner EA and Bonomo RA: Carbapenem-resistant Enterobacteriaceae: A review of treatment and outcomes. Diagn Microbiol Infect Dis 75: 115-120, 2013.

3. Qureshi ZA, Paterson DL, Potoski BA, Kilayko MC, Sandovsky G, Sordillo E, Polsky B, Adams-Haduch JM and Doi Y: Treatment outcome of bacteremia due to KPC-producing Klebsiella pneumoniae: Superiority of combination antimicrobial regimens. Antimicrob Agents Chemother 56: 2108-2113, 2012.

4. Borer A, Saidel-Odes L, Riesenberg K, Eskira S, Peled N, Nativ R, Schlaeffer F and Sherf M: Attributable mortality rate for carbapenem-resistant Klebsiella pneumoniae bacteremia. Infect Control Hosp Epidemiol 30: 972-976, 2009.

5. Carmeli Y, Akova M, Cornaglia G, Daikos GL, Garau J, Harbarth S, Rossolini GM, Souli M and Giamarellou H: Controlling the spread of carbapenemase-producing Gram-negatives: Therapeutic approach and infection control. Clin Microbiol Infect 16: 102-111, 2010.

6. Lee GC and Burgess DS: Treatment of Klebsiella pneumoniae carbapenemase (KPC) infections: A review of published case series and case reports. Ann Clin Microbiol Antimicrob 11: 32, 2012.

7. Ni W, Wei C, Zhou C, Zhao J, Liang B, Cui J, Wang R and Liu Y: Tigecycline-amikacin combination effectively suppresses the selection of resistance in clinical isolates of KPC-producing Klebsiella pneumoniae. Front Microbiol 7: 1304, 2016.

8. Lewis RE: Using carbapenems for carbapenem-resistant Klebsiella pneumoniae - are we flogging a dead (work) horse antibiotic? Virulence 3: 1-2, 2016.

9. Daikos GL and Markogiannakis A: Carbapenemase-producing Klebsiella pneumoniae: (When) might we still consider treating with carbapenems? Clin Microbiol Infect 17: 1135-1141, 2011.

10. Zarkotou O, Pournaras S, Tselioti P, Dragoumanos V, Pitiriga V, Ranellou K, Prekates A, Themeli-Digalaki K and Tsakris A: Predictors of mortality in patients with bloodstream infections caused by KPC-producing Klebsiella pneumoniae and impact of appropriate antimicrobial treatment. Clin Microbiol Infect 17: 1798-1803, 2011.

11. Pournaras S, Vrioni G, Neou E, Dendrinos J, Dimitroulia E, Poulou A and Tsakris A: Activity of tigecycline alone and in combination with colistin and meropenem against Klebsiella pneumoniae carbapenemase (KPC)-producing Enterobacteriaceae strains by time-kill assay. Int J Antimicrob Agents 37: 244-247, 2011.
12. Bratu S, Landman D, Haag R, Recco R, Eramo A, Alam M and Quale J: Rapid spread of carbapenem-resistant Klebsiella pneumoniae in New York City: A new threat to our antibiotic armamentarium. Arch Intern Med 165: 1430-1435, 2005.

13. Gasink LB, Edelstein PH, Lautenbach E, Synnestvedt M and Fishman NO: Risk factors and clinical impact of Klebsiella pneumoniae carbapenemase-producing K. pneumoniae. Infect Control Hosp Epidemiol 30: 1180-1185, 2009.

14. Marchaim D, Chopra T, Perez F, Hayakawa K, Lephart PR, Bheemreddy S, Blunden C, Hujer AM, Rudin S, Shango M, et al: Outcomes and genetic relatedness of carbapenem-resistant enterobacteriaceae at Detroit medical center. Infect Control Hosp Epidemiol 32: 861-871, 2011.

15. Hirsch EB and Tam VH: Detection and treatment options for Klebsiella pneumoniae carbapenemases (KPCs): An emerging cause of multidrug-resistant infection. J Antimicrob Chemother 65: 1119-1125, 2010.

16. Gonzalez-Padilla M, Torre-Cisneros J, Rivera-Espinar F, Pontes-Moreno A, López-Cerero L, Pascual A, Natera C, Rodriguez M, Salcedo I, Rodriguez-López F, et al: Gentamicin therapy for sepsis due to carbapenem-resistant and colistin-resistant Klebsiella pneumoniae. J Antimicrob Chemother 70: 905-913, 2015.

17. Tumbarello M, Viale P, Viscoli C, Trecarichi EM, Tumietto F, Marchese A, Spanu T, Ambretti S, Ginocchio F, Cristini F, et al: Predictors of mortality in bloodstream infections caused by Klebsiella pneumoniae carbapenemase-producing $K$. pneumoniae: Importance of combinational therapy. Clin Infect Dis 55: 943-950, 2012.

18. Qureshi ZA, Paterson DL, Peleg AY, Adams-Haduch JM, Shutt KA, Pakstis DL, Sordillo E, Polsky B, Sandkovsky G, Bhussar MK and Doi Y: Clinical characteristics of bacteraemia caused by extended-spectrum $\beta$-lactamase-producing Enterobacteriaceae in the era of CTX-M-type and KPC-type $\beta$-lactamases. Clin Microbiol Infect 18: 887-893, 2012.

19. Li Y, Zheng B, Li Y, Zhu S, Xue F and Liu J: Antimicrobial susceptibility and molecular mechanisms of fosfomycin resistance in clinical Escherichia coli isolates in mainland China. PLoS One $10: \mathrm{e} 0135269,2015$.

20. Raz R: Fosfomycin: An old - new antibiotic. Clin Microbiol Infect 18: 4-7, 2012.

21. Brown ED, Vivas EI, Walsh CT and Kolter R: MurA (MurZ), the enzyme that catalyzes the first committed step in peptidoglycan biosynthesis, is essential in Escherichia coli. J Bacteriol 177: 4194-4197, 1995.

22. Yılmaz N, A ğuş N, Bayram A, Şamlığlu P, Şirin MC, Derici YK and Hanc1 SY: Antimicrobial susceptibilities of Escherichia coli isolates as agents of community-acquired urinary tract infection (2008-2014). Turk J Urol 42: 32-36, 2016. 
23. Falagas ME, Kastoris AC, Kapaskelis AM and Karageorgopoulos DE: Fosfomycin for the treatment of multidrug-resistant, including extended-spectrum beta-lactamase producing, Enterobacteriaceae infections: A systematic review. Lancet Infect Dis 10: 43-50, 2010.

24. Roussos N, Karageorgopoulos DE, Samonis G and Falagas ME: Clinical significance of the pharmacokinetic and pharmacodynamic characteristics of fosfomycin for the treatment of patients with systemic infections. Int J Antimicrob Agents 34: 506-515, 2009.
25. Cho YH, Jung SI, Chung HS, Yu HS, Hwang EC, Kim SO, Kang TW, Kwon DD and Park K: Antimicrobial susceptibilities of extended-spectrum beta-lactamase-producing Escherichia coli and Klebsiella pneumoniae in health care-associated urinary tract infection: focus on susceptibility to fosfomycin. Int Urol Nephrol 47: 1059-1066, 2014.

26. Liu CY, Lai CC, Lee MR, Lee YC, Huang YT, Liao CH and Hsueh PR: Clinical characteristics of infections caused by Tsukamurella spp. and antimicrobial susceptibilities of the isolates. Int J Antimicrob Agents 38: 534-537, 2011. 